\title{
Do Multiple Isolated Vertebral Thoracolumbar Transverse Process Fractures Increase the Risk of Ligamentous Injury and Surgical Intervention in the Setting of Trauma?
}

\author{
Nickalus Khan', Jonathan Reding1, Matthew Gilbert², Michael S. Muhlbauer ${ }^{1,3}$, \\ L. Madison Michael ${ }^{1,3^{*}}$ \\ ${ }^{1}$ Department of Neurosurgery, University of Tennessee Health Science Center, Memphis, USA \\ ${ }^{2}$ College of Medicine, University of Tennessee Health Science Center, Memphis, USA \\ ${ }^{3}$ Semmes-Murphey Neurologic \& Spine Institute, Memphis, USA \\ Email: ^mmichael@semmes-murphey.com
}

How to cite this paper: Khan, N., Reding, J., Gilbert, M., Muhlbauer, M.S. and Michael, L.M. (2018) Do Multiple Isolated Vertebral Thoracolumbar Transverse Process Fractures Increase the Risk of Ligamentous Injury and Surgical Intervention in the Setting of Trauma? Open Journal of Modern Neurosurgery, 8, 77-83.

https://doi.org/10.4236/ojmn.2018.81006

Received: December 16, 2017

Accepted: January 15, 2018

Published: January 18, 2018

Copyright $\odot 2018$ by authors and Scientific Research Publishing Inc. This work is licensed under the Creative Commons Attribution International License (CC BY 4.0).

http://creativecommons.org/licenses/by/4.0/

\begin{abstract}
Background: Isolated thoracolumbar vertebral transverse process fractures (TPF) are often considered a stable injury. However, the use of advanced imaging such as magnetic resonance imaging and spine specialist consultation are often ordered as part of the routine workup of these fractures. The routine ordering of advanced imaging, spine specialist consultation, and delayed mobilization causes unnecessary economic and clinical burdens to patients and the overall healthcare system. Purpose: To determine if a higher number of isolated TPFs (iTPFs) lead to an increase in ligamentous injury to the spine, and whether ligamentous injury_if present-requires surgical intervention. Methods: The retrospective review was performed from 2009 to 2015, using a surgical trauma database to identify patients with greater than 3 isolated TPF (iTPF) to determine if iTPF leads to an increase in ligamentous injury to the spine and if this increase leads to increased surgical intervention. Results: A total of 102 patients were identified with complete follow up at $6-8$ weeks post injury. The majority of the included patients suffered from blunt trauma. There was a small rate of ligamentous injury $(n=7,7 \%)$ that did not require additional treatment. None of the fractures included were considered unstable. None of the patients included required surgical intervention during their hospital visit or in follow up visits. Conclusion: iTPFs are a stable injury to the thoracolumbar spine. There is a small rate of associated ligamentous injury that does not change the management or require further interventions. Thoracolumbar iTPFs do not automatically need spine specialist consultation and advanced imaging techniques.
\end{abstract}




\section{Keywords}

Transverse Process Fracture, Ligamentous Injury, Imaging, Spine Service Consultation

\section{Background}

Vertebral transverse process fractures (TPFs) in the thoracic and lumbar spine commonly occur in blunt trauma. TPFs have frequently been thought of as minor compared with pedicle, laminar, or vertebral body fractures [1]. TPFs can occur in isolation (iTPF) or in association with other spine fractures (aTPF). Approximately $10 \%$ of all major blunt truncal trauma patients have 1 or more TPFs [2] [3] [4]. A retrospective series of 191 patients from New Zealand compared iTPF with non-transverse process fractures and found a significant association of blunt abdominal injuries with iTPFs compared with other vertebral fractures [1]. This was confirmed by Patten et al. in a series of 536 patients undergoing helical computed tomography (CT) after blunt abdominal trauma [4]. Starks et al. showed a unique association of L5 TPFs in conjunction with pelvic fractures. Additionally, TPFs of L5 are associated with more unstable pelvic fractures than those not associated with L5 TPF [5].

While it is well understood that TPFs are associated with thoracic, abdominal and unstable pelvic injuries, it has been unclear in the past how seriously TPF should be taken when considering spinal stability. Spine specialist consultation historically was ordered for TPFs when X-ray was the main modality of spinal imaging so as to rule out unstable spine injuries [2]. However, with the increased use of whole body CT scanning after trauma, it is easier to rule out these significant spinal injuries and the need for specialist consultation in patients with TPF has been questioned [2] [3] [4].

At our institution, 1 or 2 iTPFs of the thoracic and lumbar spine do not receive specialist consultation and are treated conservatively with pain control and physical therapy. However, 3 or more iTPFs receive consultation to the neurosurgical or orthopedic services covering spine that day. The reason for this policy is a concern that those patients with 3 or more iTPF are associated with a higher force injury and with a suspected higher chance of ligamentous injury. These patients then receive a magnetic resonance image (MRI) through the region of the spine with the fracture and a decision is made whether or not the patient needs conservative or surgical management of their fractures.

Over the years this process has continued despite what seems to be a majority of these MRIs showing stability of the spine. The current study seeks to review this practice to see, if a higher number of iTPFs lead to an increase in ligamentous injury to the spine. The primary research question of this study is: "Does a higher number of iTPFs lead to an increase in ligamentous injury to the spine?" The secondary research question is: "If there is ligamentous injury, does it re- 
quire surgical intervention?"

Specialist consultation may not be warranted in patients with multiple iTPFs. The outcomes of this study could decrease the use of MRI scanning and likely decrease the length-of-stay of these patients, thereby improving patient care. This study examines cohort of patients with iTPFs from a 353 bed Level 1 trauma center (Regional One Medical Center, Memphis, TN) that has 13,075 admissions and 43,308 emergency room visits per annum.

\section{Methods}

After receiving IRB approval from the University of Tennessee (IRB \# 14-03444-XM), a single-center retrospective chart review was performed from January through March of 2015. The electronic health record was scanned to identify patients who sustained 3 or more iTPFs between 2009 and 2015 at a local Level 1 Trauma Center (Regional One Medical Center, Memphis, TN). Patient demographics, mechanism of injury, associated injuries, presence of ligamentous injury on MRI, need for surgical intervention, and neurological exam were recorded. Neurological examination was graded based on the American Spinal Injury Association (ASIA) impairment scale [6].

Each patient upon arrival to the hospital underwent a full body, helical CT scan with $5 \mathrm{~mm}$ cuts that included axial, sagittal, and coronal reconstructions. Those patients with 3 or more iTPFs of the thoracic and lumbar spine received a specialist consultation, and then underwent a 3Tesla MRI scan through the region of the spine with the fracture. The MRI included T1, T2, and Short TI Inversion Recovery (STIR) imaging modalities with axial and sagittal reconstructions.

Inclusion criteria for the study were 3 or more isolated transverse process fractures in the thoracic or lumbar spine. Exclusion criteria included associated thoracic or lumbar spine fractures, age $<16$, and contraindication to a MRI scan.

\section{Statistical Analysis}

All statistics were calculated using SPSS v.21 (IBM, Aramonk, NY). Significant values were considered to be $\mathrm{p}<0.05$. Mean values are presented with \pm standard deviations.

\section{Results}

A total of 102 patients were identified after retrospective chart review. The average age was $41 \pm 15$ years with a minimum of 17 years and a maximum of 81 years. There was an average of $4.8 \pm 2.2$ iTPFs per patient. The mechanism of injury was largely motor vehicle crash (MVC) followed by penetrating injury (Figure 1, Table 1 ). The majority of TPFs were lumbar $(n=69,68 \%)$, followed by thoracic $(\mathrm{n}=18,18 \%)$. Figure 2 shows the total number of TPFs by vertebral level.

The amount of ligamentous injury was minimal $(\mathrm{n}=7,7 \%)$ and the majority 


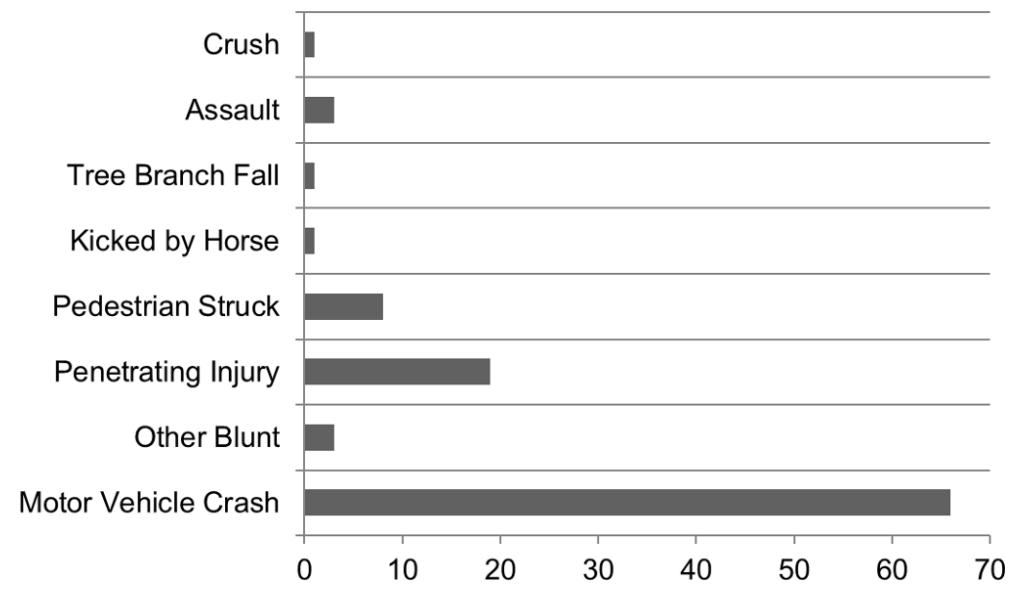

Figure 1. Causes of injury responsible for iTPFs.

Table 1. Mechanism of Injury's responsible for iTPFs.

\begin{tabular}{ccc}
\hline Mechanism & Frequency (No.) & Percent \\
\hline MVC & 66 & 64.7 \\
Blunt & 3 & 2.9 \\
Penetrating & 19 & 18.6 \\
Pedestrian Struck & 8 & 7.8 \\
Kicked by Horse & 1 & 1.0 \\
Tree Branch Fall & 1 & 1.0 \\
Assault & 3 & 2.9 \\
Crush & 1 & 1.0 \\
Total & 102 & 100.0 \\
\hline
\end{tabular}

Abbreviations: $\mathrm{iTPF}=$ isolated fractures of vertebral transverse processes; $\mathrm{MVC}=$ motor vehicle crash.

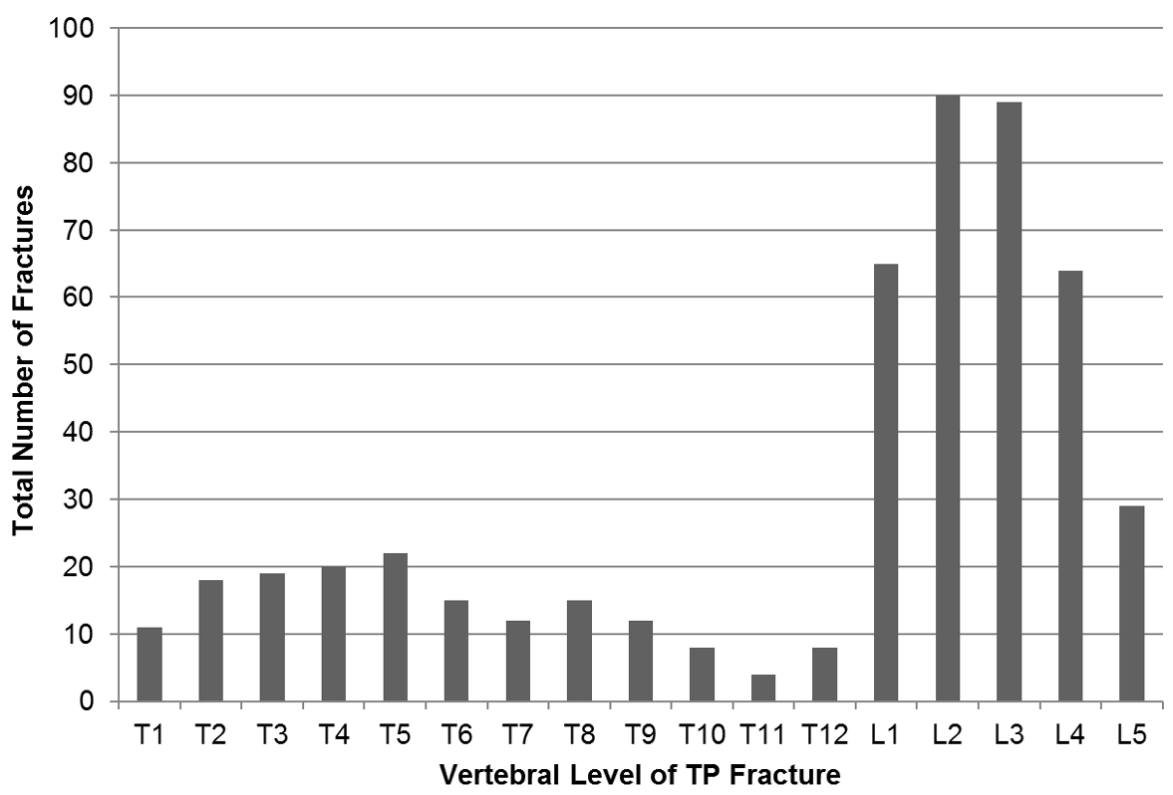

Figure 2. Total count of TPFs from all patients stratified by spinal level. The majority of TPFs were located in the upper to mid lumbar spine. 
of patients were neurologically intact $(n=100,98 \%)$. The small number $(n=2$, $2 \%)$ of patients with a neurological deficit had proximal leg weakness that improved within a day after their injury and was likely secondary to effort and pain limitation related to the back pain associated with their injury. The majority of associated injuries were pulmonary $(n=29,28 \%)$, followed by abdominal $(n=7$, $7 \%)$ and sacral injuries $(n=9,9 \%)$. Of the 102 patients seen, 40 (40\%) were treated in a brace. None of the patients in this series required surgical intervention for iTPFs during their hospital visit and follow-up visits. There was complete follow-up on all of the patients included in this study.

\section{Discussion}

Spine service consultation and advanced imaging continues to be ordered with trauma patients who have iTPFs. This continues despite an increasing amount of literature that supports conservative measures for these fractures without specialist review [2] [3]. The stability of isolated transverse process fractures has been reported as far back as Denis' seminal work on “The 3 column spine and its significance in the classification of acute thoracolumbar spinal injuries" [7]. In this paper, TPFs were categorized as a stable minor fracture of posterior column. To our knowledge, this has never been confirmed using advanced imaging techniques, such as MRI. By using MRI, we have been able to confirm what the previous literature supported. None of the patients in the current study had an unstable injury to the spine or required surgical intervention. Using the STIR sequence on MRI, a number of patients had confirmed ligamentous injury of the posterior column, the most significant of which was a torn interspinous ligament and ligamentum flavum. However, it was much more frequent for patients to have mild-to-moderate stretching of the interspinous ligament with edema noted on the MRI. These injuries are all defined as stable spinal injuries [7].

The extensive use of spine specialist consultation, advanced imaging techniques, and bracing is not without cost. The average total cost for a non-contrast lumbar spine MRI is in the range of $\$ 1000$ [8] and the cost of an average TSLO brace is approximately $\$ 200$ [9]. The cost of specialist consultation is variable and the additional length of stay, while obtaining an MRI and brace fitting certainly adds to the overall cost of care. Using a conservative estimate, including just the MRI and brace (excluding consultation costs, hospital stay costs, follow up visit costs) in this series of patients, our local health care system spent approximately $\$ 110,000$ on arguably unnecessary medical care.

All of the patients in our study had a normal neurological examination. A small number, however, did have proximal leg weakness that improved over the first few days after injury. This weakness is presumed to be because of the back pain associated with the fracture and muscles strain/injury, as well as psoas muscle injury associated with iTPFs. Early mobilization, physical therapy, and appropriate analgesia are critical to getting these patients back on their feet, pain 
controlled, and out of the hospital. Unfortunately, early mobilization is often hindered by these patients being unnecessarily placed on strict spine precautions before a spine specialist can clear their spine [2].

The question still remains about whether or not these data can be translated to cervical transverse process fractures. While this was not reviewed in the current paper, there is good evidence to support that cervical iTPFs are a stable injury. However, cervical spine iTPFs have a high association with vertebral artery injuries and must be ruled out by either $\mathrm{CT}$ angiography or digital subtraction angiography [10] [11].

As seen in other studies with TPFs, the current study saw a large number of thoracic, abdominal, and pelvic injuries associated with iTPFs. This seems to be the most important association with iTPFs, as opposed to the concern for spinal instability. One should have a high suspicion for these types of injuries when iTPFs are noted after trauma, and fortunately-with the increasing use of high quality CT scanning in the setting of trauma-these associated injuries should rarely go unnoticed.

\section{Strengths and Limitations}

This study is limited by being a single-center retrospective study. The power needed to detect an operative intervention for iTPF is likely very high and this study is underpowered to detect this rare occurrence. Another limitation is possible bias from radiological interpretations of the data (i.e., multiple different radiologists interpreted the imaging studies). Lastly, the physical exams performed by the consultation services may lack reliability among different practitioners; however, nearly all of the patients in this study had an ASIA E spinal cord injury classification, leading us to believe that this type of bias was minimal. The strengths of this study include a single cohort of patients over 5 years who all received follow-up MRI scans after their injury and had complete follow-up examination.

\section{Conclusion}

While limited by the retrospective nature and design of this study, the data suggest that patients with multiple thoracolumbar iTPFs in the setting of trauma with a normal clinical neurological examination and no associated spine fractures can be treated conservatively. iTPFs do not necessarily require subspecialty surgical consultation and advanced imaging studies.

\section{Acknowledgments}

The authors wish to thank Andrew J. Gienapp (Department of Medical Education, Methodist University Hospital, Memphis, TN and Department of Neurosurgery, University of Tennessee Health Science Center, Memphis, TN) for technical and copyediting, preparation of the manuscript for publishing, and publication assistance with this manuscript. 


\section{References}

[1] Miller, C.D., Blyth, P. and Civil, I.D. (2000) Lumbar Transverse Process Fractures-A Sentinel Marker of Abdominal Organ Injuries. Injury, 31, 773-776. https://doi.org/10.1016/S0020-1383(00)00111-X

[2] Homnick, A., Lavery, R., Nicastro, O., Livingston, D.H. and Hauser, C.J. (2007) Isolated Thoracolumbar Transverse Process Fractures: Call Physical Therapy, Not Spine. Journal of Trauma and Acute Care Surgery, 63, 1292-1295. https://doi.org/10.1097/TA.0b013e31812eed3c

[3] Bradley, L.H., Paullus, W.C., Howe, J. and Litofsky, N.S. (2008) Isolated Transverse Process Fractures: Spine Service Management Not Needed. Journal of Trauma and Acute Care Surgery, 65, 832-836; Discussion 836. https://doi.org/10.1097/TA.0b013e318184d30e

[4] Patten, R.M., Gunberg, S.R. and Brandenburger, D.K. (2000) Frequency and Importance of Transverse Process Fractures in the Lumbar Vertebrae at Helical Abdominal CT in Patients with Trauma. Radiology, 215, 831-834. https://doi.org/10.1148/radiology.215.3.r00jn27831

[5] Starks, I., Frost, A., Wall, P. and Lim, J. (2011) Is a Fracture of the Transverse Process of L5 a Predictor of Pelvic Fracture Instability? The Bone \& Joint Journal, 93, 967-969. https://doi.org/10.1302/0301-620X.93B7.26772

[6] Association, A.S.I. (2000) International Standards for Neurological Classifications of Spinal Cord Injury. American Spinal Injury Association, Chicago, Ill, 1-23.

[7] Denis, F. (1983) The Three Column Spine and Its Significance in the Classification of Acute Thoracolumbar Spinal Injuries. Spine (Phila Pa 1976), 8, 817-831. https://doi.org/10.1097/00007632-198311000-00003

[8] (2016) Average Prices for MRI at HonorHealth Facilities in Phoenix. https://www.honorhealth.com/patients-visitors/average-pricing/mri-costs\#72148

[9] Amazon Extension Orthosis TLSO Back Brace, Large. https://www.amazon.com/Extension-Orthosis-TLSO-Brace-Large/dp/B006L8MSC8

[10] Schotanus, M., van Middendorp, J.J. and Hosman, A.J. (2010) Isolated Transverse Process Fractures of the Subaxial Cervical Spine: A Clinically Insignificant Injury or Not?: A Prospective, Longitudinal Analysis in a Consecutive High-Energy Blunt Trauma Population. Spine (Phila Pa 1976), 35, E965-E970. https://doi.org/10.1097/BRS.0b013e3181c9464e

[11] Woodring, J.H., Lee, C. and Duncan, V. (1993) Transverse Process Fractures of the Cervical Vertebrae: Are They Insignificant? Journal of Trauma and Acute Care Surgery, 34, 797-802. https://doi.org/10.1097/00005373-199306000-00008

\section{Abbreviations}

ASIA = American Spinal Injury Association; aTPF = vertebral transverse processe fractures in association with other spine fractures; $\mathrm{CT}=$ computed tomography; $\mathrm{iTPF}=$ isolated vertebral transverse process fractures; $\mathrm{MRI}=$ magnetic resonance image; $\mathrm{MVC}=$ motor vehicle crash; STIR $=$ Short TI Inversion Recovery; TPF $=$ vertebral transverse process fractures. 